\title{
Prevalence of work related musculoskeletal disorders in active union carpenters
}

\author{
G K Lemasters, M R Atterbury, A D Booth-Jones, A Bhattacharya, N Ollila-Glenn, \\ C Forrester, L Forst
}

University of Cincinnati, College of Medicine, Department of Environmental Health, 3233 Eden Avenue, ML-182, Cincinnati, OH 45267-0182, USA G K Lemasters A D Booth-Jones A Bhattacharya

Greater Cincinnati Occupational Health Center, 311 Howell Ave, Cincinnati, OH 45220, USA M R Atterbury N Ollila-Glenn C Forrester

University of Illinois at Chicago, $813 \mathrm{~S}$ Wolcott, 392 Chicago, IL 60612, USA L Forst

Correspondence to: Professor G Lemasters, University of Cincinnati, College of Medicine, Department of Environmental Health, 3233 Eden Avenue, ML-182, Cincinnati, OH 45267-0182, USA.

Accepted 12 January 1998

\begin{abstract}
Objectives-To determine the prevalence and risk factors for work related musculoskeletal disorders among union carpenters.

Methods-A detailed questionnaire on musculoskeletal symptoms and work history was administered to 522 carpenters. The symptom questions assessed if carpenters experienced pain, numbness, or tingling in a particular body region. A subset of this group then received a physical examination of the upper extremities and knees.
\end{abstract}

Results-The study group was primarily white $(94.9 \%)$ and male $(97.8 \%)$ with a mean age of 42.3 years. The highest prevalence of work related musculoskeletal disorders cases by carpentry specialty ranged from $20 \%-24 \%$ for those doing drywall or ceiling, finishing or framing, and the building of concrete forms. Generally, as duration of employment increased, the prevalence of symptoms increased. An adjusted logistic regression analysis showed that the group with the longest ( $\geqslant 20$ years) duration of employment in carpentry was significantly associated with work related musculoskeletal disorders of the shoulders (odds ratio (OR) $3.2,95 \%$ confidence interval $(95 \%$ CI) 1.1 to 8.9), hands or wrists(OR 3.1, 95\% CI 1.1 to 8.4), and knees (OR 3.5, 95\% CI 1.3 to 9.2). Also, analyses showed that carpenters who reported that they had little or no influence over their work schedule had significant increases of work related musculoskeletal disorders of the shoulders, hips, and knees with ORs of 1.9 (95\% CI 1.1 to 3.2), 2.9 (95\% CI 1.1 to 7.2$)$, and $2.3(95 \%$ CI 1.2 to 4.1$)$, respectively. Feeling exhausted at the end of day was also a significant risk factor for work related musculoskeletal disorders of the knee (OR 1.8, 95\% CI 1.1 to 3.1). Upper extremity disorders were the most prevalent work related musculoskeletal disorders reported among all carpenters. Drywall or ceiling activities involve a considerable amount of repetitive motion and awkward postures often with arms raised holding heavy dry walls in place, whereas form work is notable for extensive lumbar flexion and had the two highest rates of work related musculoskeletal disorders. The psychosocial element of job control was associated with both upper and lower extremity disorders. These union carpen- ters, who were relatively young, already were experiencing considerable work related physical problems.

Conclusion-This study supports the need for vigilant ergonomic intervention at job sites and early ergonomic education as an integral part of apprenticeship school training to ensure that carpenters remain fit and healthy throughout their working lifetime.

(Occup Environ Med 1998;55:421-427)

Keywords: construction; carpenters; work related musculoskeletal disorders; job stress; job control

The term work related musculoskeletal disorders is used in reference to conditions also called cumulative trauma disorder, repetitive strain injury, or overuse syndromes. These conditions involving muscles, tendons, or nerves are generally manifested by pain, discomfort, or tingling in a body region. The term work related musculoskeletal disorders is preferred, however, because of the multifactorial nature of these conditions. The vast majority of research on work related musculoskeletal disorders, including rates, identification of risk factors, clinical diagnosis, and return to work issues, has been conducted in the manufacturing or meat processing industries and among office personnel. ${ }^{1-6}$ In these occupations work activities and worksites are generally predictable and job tasks involving ergonomic risk are usually limited to one or two body areas. By contrast, employment in the construction trades entails use of multiple body regions requiring the handling of hand and power tools, constant movement in awkward positions, and repetitive, forceful use of the back and upper and lower extremities; all of which are known risk factors for work related musculoskeletal disorders. ${ }^{7-11}$ Besides these recurrent musculoskeletal stressors, construction workers often perform job tasks under adverse environmental conditions - such as inclement weather-at varying elevations, and with composition of work crews subject to frequent changes. Tasks are often performed at a maximum pace to meet construction deadlines. These conditions place the carpenter at risk of acute injuries as well as chronic musculoskeletal disorders. Finally, the phenomenon of intermittent employment with various employers from periods of days to years, adds unfamiliar work environments and work practices to this hazardous equation. Because of this unique combination of factors, 
research focused on other industries cannot be extrapolated to construction.

Hsiao and Stanevich ${ }^{12}$ with data collected from the United States Department of Labor statistics, the United States Department of Commerce, and from data collected in 1987 and 1988 from 25 states for the Bureau of Statistics' supplementary data systems showed that in $\mathbf{1 9 9 0}$ the construction industry had the highest incidence of fatalities, injuries, and illnesses per 100 full time workers in major industries. For the construction industry the rate was 14.2 compared with a rate of 13.2 , $11.6,9.6$, and 8.3 for the manufacturing, agriculture, transportation, and mining industry, respectively. Within the construction industry, 30 occupations were evaluated to prioritise jobs for injury prevention, and carpentry ranked second highest, after construction labourers.

Carpenters are involved in all phases of commercial and residential building construction, as well as in the construction of roads, bridges, and tunnels, representing numerous exposures to both chemical and physical factors. In another study evaluating 27362 members of the United States Carpenters Union who died between 1987 and 1990, significant elevated age adjusted proportionate mortalities were found for diagnoses of asbestosis, emphysema, transportation injuries, falls, and cancers of the lung, bone, and male breast. ${ }^{13}$ Thus there is a need to better understand this complex industry to target important areas of intervention.

During framing and interior finishing activities, carpenters work with tools often held overhead or below waist level, while sometimes balancing on ladders or the structural frame itself. Fabricating wooden forms for pouring concrete requires forceful use of hand held power tools, grasping heavy lumber, manual hammering, and fastening of forms. Drywall and ceiling installation requires the repetitive grasping, lifting, forceful operation of screwguns, often reaching above the shoulder or below the knee to insert screws into wood or metal studs, and carrying of gypsum boards generally weighing $5-10 \mathrm{~kg}$, but depending on thickness of the drywall they can range up to $\geqslant 40 \mathrm{~kg}$. Because of these tasks and others, carpenters experience insults to almost every body region during the performance of their jobs. In Ohio, where this study was undertaken, construction workers reported a higher rate of work related musculoskeletal disorders than all other occupational groups, with carpenters having the largest increase in lost time due to injuries of all the construction trades. ${ }^{14}$ There has been minimal research focused on work related musculoskeletal disorders in the carpentry trade, although an understanding of their rate, for subspecialities in particular, is essential in developing strategies for the prevention of work related musculoskeletal disorders. Therefore, this study was undertaken to determine the prevalence and risk factors for work related musculoskeletal disorders among currently active union carpenters.

\section{Subjects and methods} SUBJECTS

A computerised membership list comprising 2074 union carpenters was obtained from the Southwest Ohio District of Carpenters Local 126 and Local 2. From this list a systematic random sample of 660 carpenters was generated. All union members who had performed carpentry work during the previous 12 months were eligible to participate in the study. Thirty three of these 660 carpenters had either retired or left the trade for a year or longer and thus were ineligible. Of the 627 remaining active union carpenters, $522(83 \%)$ participated in the interview. Of the 105 non-participants, 37 refused to be interviewed, and 68 could not be located.

Before the telephone interview each carpenter was sent a letter explaining the purpose of the study, a coloured body region diagram, and a letter of support signed by a union official. The coloured diagram was provided to facilitate their identification of separate body regions-for example, neck versus shoulder-as well as to aid the subject in recalling the study letter and its purpose. The questionnaire was administered by telephone interview with direct entry on to a computer. The data were evaluated for consistency at the time of entry. Data analyses were performed on questionnaire information supplied by 490 of the subjects. Data from 32 subjects were not included in the analyses because they were not currently employed as carpenters or had supplied information judged to be incomplete or unreliable.

\section{QUESTIONNAIRE CONSTRUCTION}

The questionnaire was divided into five sections: demographics, symptoms and injuries, medical history, work history, and psychosocial factors. A detailed description of the development and administration of the survey instrument and a copy of it have been published elsewhere. ${ }^{15}$ To assess the presence of persistent musculoskeletal symptoms, the initial screening question was "Within the past 12 months have you experienced any recurring symptoms such as pain, aching, numbness or any other symptoms in your neck, shoulder, elbow, forearm, hand or wrist, back, hip, thigh, knee, or ankle?" Each carpenter was allowed to qualify a positive response to the occurrence of a given symptom with "burning", "pain", "numbness or tingling" or any combination of these. An affirmative response also generated a series of further questions to establish onset, quality, frequency, duration, and intensity of symptoms, and a history of injury to that body region.

In this population, injuries were suspected of being a common cause of musculoskeletal symptomatology. Consequently, another set of questions was used to characterise injury to individual body regions. Injuries included a sudden event such as a fall, an object hitting the worker, or an injury associated with motor accidents or sports activities. The intention of these questions was to exclude those people who reported a history of injury to that body 
region. The medical history established the presence of conditions diagnosed by a physician possibly related to development of work related musculoskeletal disorders. These conditions could be either other risk factors - such as diabetes mellitus, rheumatoid arthritis, hypothyroidism, or conditions resulting in similar symptoms - for example, herniated cervical disc.

Characterising carpentry tasks associated with musculoskeletal stressors was complex due to the wide variety of tasks performed, and job rotations among specialties. Hence, duration of employment in the carpentry trade was used as the primary surrogate measure of exposure to ergonomic stressors. To find if a subspecialty - for example, finishing or formwas more ergonomically hazardous than another for a particular body area, carpenters were queried on current participation in individual specialities.

Job related psychosocial stress and work organisation factors were also of concern as risk factors for work related musculoskeletal disorders have not been well characterised in carpenters. It has been hypothesised that a worker is at greatest risk when the worker's control over the task is low and the demands of a job are high. ${ }^{16-18}$ Therefore, questions that considered these two factors were included from another questionnaire shown to have good reliability. ${ }^{19}$ The first set of psychosocial questions asked about the level of job control the person had over the amount of work, availability of materials, policies and procedures, pace of work, quality of work, and their scheduled hours. Demands of the job were considered with questions related to how fast and hard they had to work, how physically exhausted the carpenters felt, the extent of their work loads, and job satisfaction. Before administration to the study group, the questionnaire was pretested on a small sample (about 10 subjects) and pilot tested with 50 carpenters belonging to a union in another city. Final changes were then made before its implementation.

OUTCOME DEFINITION

A person was defined as having a work related musculoskeletal disorder with the following criteria: $(a)$ onset of symptoms occurred after starting work as a carpenter; (b) symptoms occurred at least once a week (frequency); (c) or lasted for one week (duration), and (d) having no history of injury to that body region. Investigators from the National Institute for Occupational Safety and Health (NIOSH) used a similar outcome definition. ${ }^{6}$ Because this population of workers are generally stoic and accustomed to considerable physical discomfort associated with their work, a severity factor was not used for the case definition.

SAMPLE SIZE AND ANALYSIS STRATEGIES

Back and knee joint injury were the two most prevalent work related musculoskeletal disorders reported through the Ohio workers' compensation system with knee being the less common and most conservative rate chosen for sample size calculations. The prevalence of knee joint damage in a potentially more highly exposed group - that is, floor and foundation layers-may range between $20 \%$ and $40 \%$ compared with millwrights, whose reported knee symptoms were between $5 \%$ and $25 \% .^{20}$ Therefore, it was determined that a sample size of about 133 was needed in each group with calculations based on a somewhat arbitrary, but relatively conservative, decision to detect a twofold difference between a rate of $15 \%$ compared with a higher risk group of $30 \%$ with $80 \%$ power at a significant level of 0.05 , with a two sided test. ${ }^{21}$

Analyses included descriptive statistics as well as unadjusted and adjusted odds ratios (ORs) calculated with a multivariate logistic regression analysis. The primary analyses used duration of employment in carpentry as the surrogate measure of ergonomic risk in the carpentry trade. The first step was to examine other possible risk factors with univariate techniques. These factors included age in years; age as a categorical variable; current weight; a previous medical condition; participation in a racquet or ball sport or fishing; and the psychosocial variables previously described. A matrix of possible covariates versus each body region was constructed. This step facilitated choosing a "common" pool of covariates that had moderate to high levels of association for most body regions. These variables were then included in the initial variable modelling step for each body region. Harrell et a ${ }^{2}$ suggests that the number of predictors should be no greater than the number of cases divided by 10 . Hence, six variables were chosen for inclusion: duration of employment as a carpenter defined by three categories: $<10$ years, $10-19$ years, and $\geqslant 20$ years, age (in years or defined categorically as $1=\geqslant 41$ years, $0=\leqslant 40$ years), smoking ( $1=$ current smoker, $0=$ non-smoker), previous adverse health condition (diabetes, gout, rheumatoid arthritis, lupus as $1=y e s, 0=$ no) feeling exhausted at the end of the day $(1=$ often or very often, $0=$ otherwise), and influence over their work schedule ( $1=$ no to moderate influence, $0=$ otherwise). The significance of each variable was confirmed by examining the Wald statistic and by comparing the estimated coefficient with the coefficient obtained in the univariate modelling. Variables that were not significant were removed, and the model was retested. The new model was compared with the old by the likelihood ratio test.

To assess the possible confounding effects of age, a change in the duration coefficient was tested with and without the age variable. As there was a small change in these estimates for some of the body regions, and as age is a known risk factor, age was included in all models to control for its potential confounding effects. Biological plausibility and a $p$ value of $5 \%$ was used to determine the possible importance of interaction in the model. Interactions were examined by adding the second order interaction terms to the main effects model. Differences in the log likelihood statistics between the main effects only and the main effects plus 
interactions models were used to test significance of the interaction terms, and none were found.

VALIDITY AND RELIABILITY ASSESSMENTS

To validate the prevalence of work related musculoskeletal disorders as reported on the questionnaire interview, a subset of carpenters was recruited to receive a physical examination, within about three weeks after completing the initial questionnaire. Subject recruitment for this substudy began and ended before completion of all questionnaire interviews. Eligibility for participation in the physical examination required that cases reported continued moderate or greater discomfort with no new injury to that body region. At the time of recruitment for the validity study, there were 38 hand or wrist, 31 shoulder, and 31 knee cases identified. Of the 100 cases identified and recruited, 23 reported no longer having pain and hence were ineligible, and 11 had work schedule conflicts or refused. At the time of recruitment there were 134 totally asymptomatic carpenters; 60 were randomly selected and recruited until a sample of 35 agreed to participate. The 25 non-participants included 10 refusals, seven with work schedule conflicts, five who were never reached, two not currently doing carpentry, and one with a recent injury.

Standardised musculoskeletal examinations of the neck, upper extremities, and knees were performed by two physician examiners who were blinded to the case-control status of the carpenters, their symptoms, and their work history. The upper extremity portion of the examination included range of motion, passive and resisted movements, and specific diagnostic manoeuvres for biceps tendinitis, thoracic outlet syndrome, carpal tunnel syndrome, and de Quervain's tenosynovitis. The knee was examined for evidence of degenerative joint disease by either the presence of pain with patellar compression or range of motion, joint line tenderness, or evidence of effusion. One electromyographer blinded to case-control status and symptoms results from the physical examination, or work history, performed unilateral nerve conduction studies both on hand or wrist cases and asymptomatic carpenters by standard techniques reported elsewhere. ${ }^{9}$ Two hand or wrist cases did not tolerate complete nerve conduction testing, and therefore, were not included in that part of the analysis.

Table 1 Prevalence of work related musculoskeletal disorders (WMDs) by body region by primary carpentry subspecialty during past 12 months $(n=489) \dagger$

\begin{tabular}{|c|c|c|c|c|c|c|c|c|}
\hline \multirow[b]{2}{*}{ Body region } & \multicolumn{2}{|c|}{ Form $(n=105)$} & \multicolumn{2}{|c|}{$\begin{array}{l}\text { Finishing or } \\
\text { framing } \\
(n=132)\end{array}$} & \multicolumn{2}{|c|}{$\begin{array}{l}\text { Drywall or } \\
\text { ceiling }(n=113)\end{array}$} & \multicolumn{2}{|c|}{ Other $(n=139)$} \\
\hline & $n^{\star}$ & $\%$ & $n^{\star}$ & $\%$ & $n^{\star}$ & $\%$ & $n^{\star}$ & $\%$ \\
\hline Neck & 8 & 7.6 & 12 & 9.1 & 13 & 11.5 & 12 & 8.6 \\
\hline Shoulders & 25 & 23.8 & 21 & 15.9 & 20 & 17.7 & 24 & 17.3 \\
\hline Elbows & 24 & 22.9 & 22 & 16.7 & 20 & 17.7 & 26 & 18.7 \\
\hline Hand or wrist & 19 & 18.1 & 28 & 21.2 & 23 & 20.4 & 22 & 15.8 \\
\hline Back & 18 & 17.1 & 22 & 16.7 & 12 & 10.6 & 25 & 18.0 \\
\hline Hip & 11 & 10.5 & 8 & 6.1 & 7 & 6.2 & 8 & 5.8 \\
\hline Knees & 15 & 14.3 & 19 & 14.4 & 21 & 18.6 & 20 & 14.4 \\
\hline Ankles & 6 & 3.8 & 2 & 1.5 & 8 & 7.1 & 5 & 3.6 \\
\hline
\end{tabular}

^People may have reported more than one WMD; so will not be equal to total in ( ). †Missing information for one person.
The reliability of a questionnaire is the extent to which results obtained by a procedure, in this case telephone interviews, can be replicated. The reliability of the questionnaire was assessed with the test-retest method. Portions of the questionnaire were readministered to about $9 \% \quad(n=49)$ of carpenters randomly selected from the interviewed group. The reinterview (retest) occurred between one and three weeks after completion of the original interview. The areas considered in the reliability assessment included recall of previous medical conditions, previous injuries, work histories, and reporting of symptoms. Details of the reliability study are available elsewhere. ${ }^{23}$

\section{Results}

The study group was primarily white $(94.9 \%)$, and male $(97.8 \%)$ with a mean (SD) age of 42.3 (10.6) years. Mean (SD) duration of employment as a carpenter was 18 (10.3) years. The prevalence of work related musculoskeletal disorders was examined first by the subspecialty that the subjects reported doing most over the previous 12 months. These subspecialties were defined as concrete form $(n=105)$, framing and finishing $(n=132)$, drywall and ceiling $(n=113)$, and other $(n=139)$. The other category included supervisory $(n=37)$, scaffolding $(n=31)$, flooring $(n=22)$, welding $(\mathrm{n}=15)$, and a miscellaneous group $(n=34)$. In this analysis, a carpenter may have reported more than one work related musculoskeletal disorder. All specialties had a high rate of these, but the specialty with the highest was form work $(118 \%)$, followed by drywall and ceiling $(110 \%)$, framing and finishing $(102 \%)$, and other $(102 \%)$. Carpenters involved in form work reported work related musculoskeletal disorders primarily of the shoulders $(23.8 \%)$ and elbows $(22.9 \%)$, whereas those doing framing or finishing and drywalling or ceiling activities had the highest rates for the hands and wrists $(20 \%-21 \%$, table 1$)$. Those in the other category had work related musculoskeletal disorders of the elbows $(18.7 \%)$ and back $(18 \%)$.

Total duration of employment as a carpenter was analysed categorically; those working in carpentry for $<10$ years $(n=113)$ made up the comparison group for both the medium duration group of between 10 and 19 years $(n=179)$, and the long duration group of $\geqslant 20$ years $(n=198)$. Generally, as duration of employment increased, the prevalence of symptoms also increased (table 2). The unadjusted ORs for work related musculoskeletal disorders of the hand or wrist and the shoulder were significantly increased (more than twofold) in the medium and longest duration groups. For the medium duration group, the ORs were increased for seven of the eight body regions and for all in the longest duration group. Because of a concern that work related musculoskeletal disorders might be initiated earlier in a carpenter's career, a second analysis investigated the following categories: baseline group $<5$ years $(n=46)$, medium duration group 5-19 years $(n=246)$, and long duration group $\geqslant 20$ years $(n=198)$. The findings were 
Table 2 Work related musculoskeletal disorders (WMDs) by body region and duration of employment as a carpenter: unadjusted OR (95\% CIs) $(n=490)$

\begin{tabular}{|c|c|c|c|c|c|c|}
\hline \multirow[b]{2}{*}{ Body region } & \multicolumn{3}{|c|}{$\begin{array}{l}\text { OR }(95 \% C I)+(10 \text { and }<20 y) \\
(n=179)\end{array}$} & \multicolumn{3}{|c|}{ OR $(95 \% C I)+(\geqslant 20 y)(n=198$} \\
\hline & $n^{\star}$ & $\%$ & OR $(95 \% C I)$ & $n^{\star}$ & $\%$ & OR $(95 \% C I)$ \\
\hline Neck & 13 & 7.3 & $1.0(0.4$ to 2.6$)$ & 24 & 12.1 & $1.8(0.8$ to 4.2$)$ \\
\hline Shoulders & 32 & 17.9 & 2.5 (1.2 to 5.5$)$ & 49 & 24.7 & $3.8(1.8$ to 8.1$)$ \\
\hline Elbows & 38 & 21.2 & $1.6(0.9$ to 3.1$)$ & 38 & 19.2 & $1.4(0.8$ to 2.7$)$ \\
\hline Hand and wrist & 36 & 20.1 & $2.3(1.1$ to 4.8$)$ & 45 & 22.7 & $2.7(1.4$ to 5.5$)$ \\
\hline Back & 31 & 17.3 & $1.4(0.7$ to 2.7$)$ & 31 & 15.7 & $1.2(0.6$ to 2.4$)$ \\
\hline Hips & 12 & 6.7 & $2.0(0.6$ to 6.2$)$ & 18 & 9.1 & $2.7(0.9$ to 8.3$)$ \\
\hline Knees & 29 & 16.2 & $1.6(0.8$ to 3.3$)$ & 34 & 17.2 & $1.8(0.9$ to 3.5$)$ \\
\hline Ankles & 4 & 2.2 & $0.4(0.1$ to 1.5$)$ & 9 & 4.5 & $0.9(0.3$ to 2.5$)$ \\
\hline
\end{tabular}

^People may have reported more than one WMD; so will not be equal to total in ( ). †Comparison group is $<10$ y duration $(n=113)$.

even more pronounced but the confidence intervals were less stable. Work related musculoskeletal disorders of the shoulder were significant for both the medium (OR 8.7, 95\% CI 11.2 to 65.2 ) and long duration (OR 14.8, $95 \%$ CI 2.0 to 110.2 ) groups. Also of interest was the slightly decreasing trend found for work related musculoskeletal disorders of the back for the medium (OR 3.0, 95\% CI 0.9 to 10.2) compared with the long (OR $2.7,95 \% \mathrm{CI}$ 0.8 to 9.1 ) duration groups. Work related musculoskeletal disorders of the back showed a slight decrease in the OR as the duration of carpentry increased. When the baseline comparison group was changed from $<10$ to $<5$, however, the OR more than doubled from 1.4 to 3.0 for the medium group and from 1.2 to 2.7 for the long duration groups.

The final analysis was a multivariate logistic regression model examining six main effects by body area including duration as carpenter, smoking status, feeling exhausted at end of the day, having minimal influence over the schedule, and having had a previous illness (table 3). Duration of employment as a carpenter for $\geqslant 20$ years was significantly associated with a work related musculoskeletal disorder of the shoulder (OR 3.2, 95\% CI 1.1 to 8.9 ), hand or wrist (OR 3.1, 95\% CI 1.1 to 8.4), and knee (OR 3.5, 95\% CI 1.3 to 9.2 ). The risk factor of having minimal influence over the work schedule also was associated with work related musculoskeletal disorders of the shoulders, hips, and knees with ORs of 1.9, 2.9, and 2.3, respectively. Also, feeling exhausted at the end of the day was significant for work related musculoskeletal disorders of the knee, and having a previous illness was significant for disorders of the hip. Age was not significant when included in the full multivariate model as a main or interaction effect, but it did show some confounding effects with job duration. As might be expected with a high correlation between age and job duration, if age was substituted for duration in the model, age became significant for work related musculoskeletal disorders of both shoulders and hands or wrists. The magnitude of the age effect, however, was not as strong as the effect of duration in these instances.

\section{VALIDITY AND RELIABILITY RESULTS}

The overall findings of the physical examinations were largely confirmatory for the questionnaire data (table 4). Shoulder cases identified from histories on the questionnaires were significantly associated with findings on the physical examinations for rotator cuff tendinitis, $78.9 \%$, compared with $5.3 \%$ for the asymptomatic controls $(p<0.005)$. Prepatellar and infrapatellar tendinitis were similar between groups but degenerative joint disease was more than sixfold higher in the cases $(38.1 \%)$ than in the controls $(5.7 \%)$. Results of the physical examination of the hands and wrists showed that carpal tunnel syndrome was significantly more common among cases $(48.0 \%)$ than controls $(14.3 \%)$, and $78.0 \%$ of the hand or wrist cases met the criteria for a median mononeuropathy of the wrist - that is, symptoms of carpal tunnel syndrome. ${ }^{9}$ In summary, cases with disorders of the knee, shoulder, and hand or wrist were significantly associated with positive findings on physical examination that included nerve conduction studies. Of importance, however, is that median mononeuropathy was also found among $34 \%$ of the asymptomatic controls indicating a high degree of pathology among all carpenters.

The reliability assessment was also supportive of the study findings. ${ }^{23}$ The agreements between the test and retest interviews ranged from $75.5 \%$ to $95.7 \%$. The agreement for the musculoskeletal symptom was $75.8 \%(\kappa=0.5)$. Thirty seven of the 49 reinterviewed subjects responded identically. Eight changed from yes of having a symptom to no on the retest and four did the reverse. Some of these differences were likely to be due to true changes in their symptoms between the initial interview and the reinterview.

Table 3 Risk factors * significantly associated with prevalence of work related musculoskeletal disorders by body region adjusted OR (95\% CIs)

\begin{tabular}{|c|c|c|c|c|c|c|}
\hline \multirow[b]{2}{*}{ Body region } & \multicolumn{2}{|c|}{ Durations as a carpenter } & \multirow[b]{2}{*}{ Current smoker } & \multirow{2}{*}{$\begin{array}{l}\text { Exhausted end of } \\
\text { day }\end{array}$} & \multirow{2}{*}{$\begin{array}{l}\text { Minimal } \\
\text { schedule } \\
\text { influence }\end{array}$} & \multirow{2}{*}{$\begin{array}{l}\text { Previous health } \\
\text { condition }\end{array}$} \\
\hline & $\geqslant 10$ and $<20 t$ & $\geqslant 20 t$ & & & & \\
\hline Neck & $0.9(0.4$ to 2.5$)$ & $1.5(0.4$ to 5.4$)$ & $1.0(0.5$ to 2.0$)$ & $1.1(0.6$ to 2.2$)$ & $1.8(0.9$ to 3.5$)$ & $1.6(0.7$ to 3.7$)$ \\
\hline Shoulders & $2.3(1.02$ to 5.4$)$ & $3.2(1.1$ to 8.9$)$ & $1.0(0.6$ to 1.7$)$ & $1.5(0.9$ to 2.4$)$ & $1.9(1.1$ to 3.2$)$ & $0.8(0.4$ to 1.6$)$ \\
\hline Elbows & $1.7(0.8$ to 3.3$)$ & $1.7(0.7$ to 4.0$)$ & $1.7(1.0$ to 2.6$)$ & $1.4(0.9$ to 2.2$)$ & $1.6(0.9$ to 2.6$)$ & $1.2(0.6$ to 2.3$)$ \\
\hline Hand and wrist & $2.4(1.1$ to 5.3$)$ & $3.1(1.1$ to 8.4$)$ & $1.1(0.7$ to 1.7$)$ & $1.5(0.9$ to 2.5$)$ & $1.6(1.0$ to 2.7$)$ & $1.8(1.0$ to 3.4$)$ \\
\hline Back & $1.5(0.8$ to 3.1$)$ & $1.9(0.8$ to 4.6$)$ & $1.3(0.8$ to 2.2$)$ & $1.3(0.8$ to 2.1$)$ & $1.1(0.6$ to 1.8$)$ & $1.0(0.5$ to 2.0$)$ \\
\hline Hips & $1.9(0.5$ to 6.6$)$ & $2.8(0.6$ to 13.5$)$ & $1.1(0.6$ to 2.4$)$ & $1.4(0.7$ to 3.0$)$ & $2.9(1.1$ to 7.2$)$ & $2.5(1.1$ to 5.9$)$ \\
\hline Knees & $1.9(0.9$ to 4.1$)$ & 3.5 (1.3 to 9.2$)$ & $1.5(0.9$ to 2.6$)$ & $1.8(1.1$ to 3.1$)$ & $2.3(1.2$ to 4.1$)$ & $1.7(0.9$ to 3.5$)$ \\
\hline Ankles & $0.4(0.1$ to 1.8$)$ & $1.1(0.2$ to 6.7$)$ & $1.0(0.4$ to 2.5$)$ & $0.7(0.3$ to 1.9$)$ & $2.5(0.8$ to 7.8$)$ & $2.7(0.8$ to 8.7$)$ \\
\hline
\end{tabular}

* Model included age which was not significant for any outcome; ORs ranged from 0.5 to 1.02 .

†Comparison group is $<10$ y duration $(n=113)$. 
Table 4 Validation of questionnaire by physical examination and nerve conduction

\begin{tabular}{|c|c|c|c|}
\hline Body region & Examination results & $\begin{array}{l}\text { Cases } \\
n(\%)\end{array}$ & $\begin{array}{l}\text { Asymptomatic } \\
\text { controls } n(\%)\end{array}$ \\
\hline \multirow[t]{3}{*}{ Shoulder (n) } & & $19 \sqrt{2}$ & 35 \\
\hline & Rotator cuff tendinitis & $15(78.9)$ & $1(5.3)^{\star \star}$ \\
\hline & Biceps tendinitis & $1(5.3)$ & $1(5.3)$ \\
\hline \multirow[t]{5}{*}{ Hand and wrist (n) } & & 25 & 35 \\
\hline & Carpal tunnel syndrome & $12(48.9)$ & $5(14.3)^{\star}$ \\
\hline & Wrist tendinitis & $5(20.0)$ & $4(11.4)$ \\
\hline & Medium mononeuropathy $\dagger$ & $19(78.0)$ & $12(34.0)$ \\
\hline & Other $\ddagger$ & $3(12.0)$ & $2(5.7)$ \\
\hline \multirow[t]{3}{*}{ Knees (n) } & & 21 & 35 \\
\hline & Prepatellar or infrapatellar tendinitis & $1(4.8)$ & $1(5.3)$ \\
\hline & Degenerative joint disease & $8(38.1)$ & $2(5.7)^{\star \star \star}$ \\
\hline
\end{tabular}

$\star(\mathrm{p}=0.005)$ Mantel-Haenzel $\chi^{2}=8.03$

$\star \star(\mathrm{p}<0.001)$ Mantel-Haenzel $\chi^{2}=33.56$

$\star \star \star(\mathrm{p}<0.002)$ Mantel-Haenzel $\chi^{2}=9.21$.

$\dagger$ Nerve conduction test $(n=23)$.

$\ddagger$ Other includes DeQuervain’s tenosynovitis, ganglion cysts, trigger finger, joint related conditions.

$\$$ Missing data on one physical examination.

\section{Discussion}

Work related musculoskeletal disorders of the upper extremities, in particular shoulders and hands or wrists, were most prevalent among all carpenters particularly drywall or ceiling and formwork subspecialties. Drywall and ceiling installation entails repetitive, forceful use of screw guns often held in awkward positions, requiring sustained overhead reaching. Hence, the high prevalence of upper extremity work related musculoskeletal disorders is not surprising. Concrete form tasks had the highest prevalence of shoulder and elbow disorders. In another on site ergonomic study by our research team, carpenters performing ceiling, dry wall, and form tasks were evaluated. ${ }^{24}$ The most stressful postures were found to stress the neck, shoulder, elbow, and back regions.

Because heavy material handling is characteristic of carpentry, a higher prevalence of work related musculoskeletal disorders of the back was expected. Final analysis, however, showed $>80 \%$ power to detect a significant difference in back disorders even though none was found. This lower than expected prevalence of back disorders may be related to choice of jobs by carpenters prone to back disorder or to a survivor effect. Those people with back problems may have had to leave or change jobs as carpentry tasks require considerable bending and lifting. Support for this hypothesis may be provided by comparison of the baselines of the two duration groups. When the baseline was shifted from duration in carpentry of $<10$ years to $<5$ years, the OR for back disorders of the medium duration group increased from 1.4 to 3.0, respectively. This finding suggests that an increased risk of back disorders occurs early, before 10 years of employment in the trade. Further, table 2 shows that the OR decreased slightly for those with $\geqslant 20$ years in the trade rather than increasing as was expected. Thus, the lower prevalence of back disorders may be due to the increased total disability caused by back disorders leading to earlier retirement from the trade or change to a less ergonomically stressful job. For most other body areas, however, as duration of employment in carpentry increased there was an increase in work related musculoskeletal disorders.
Although age was not significantly associated with work related musculoskeletal disorders in the full multivariate model, it did function as a confounder for shoulders, knees, and back. Therefore, as a meaningful biological factor, it was included in all models for each body region. Modelling correlated time dependent variables - such as duration of employment and the subject's age..$^{25}$ If both age in years and duration of employment are treated as continuous variables and allowed to simultaneously explain the outcome, the net result may be multicollinearity causing unstable variance estimates. To control for this problem, duration of employment was treated as a categorical (dummy) variable comparing the baseline group separately with the medium and longer duration groups, and age was primarily evaluated as a continuous variable.

Psychosocial and work organisation factors have played a large part in the occurrence of upper extremity disorders among office workers. ${ }^{6}$ Few studies have investigated the role of job related psychosocial stressors and work organisation in the occurrence of work related musculoskeletal disorders, particularly in construction workers. Carpenters and other people in the building trade must work under contractor and project deadlines. Availability of materials, tools, and material handling devices are governed by the contractor. Also, the existence of multiple subcontractors is common and reduces the control a carpenter has at any one job site. It has been hypothesised that a worker is at greatest risk when control over a task is low, but the demands of a job are high. ${ }^{17}{ }^{18}$ This relation was supported with disorders of the shoulders, hips, and knees.

The physical examination and nerve conduction results supported the findings of the questionnaire. The case definition for the questionnaire data, however, included those reporting mild symptoms, and therefore, was not directly comparable with the physical examination group who had moderate to severe symptoms. The validity of the questionnaire for those reporting mild symptoms cannot be evaluated.

Finally, as with most cross sectional studies, currently employed carpenters were evaluated leading to a possible selection bias. Hence, this study may have underestimated true risk as only those currently in the trade were interviewed. A longitudinal study at time of entry into the carpentry trade is recommended to better understand risk and predictive factors related to leaving or staying in carpentry. Certainly, this study supports the need for early ergonomic training as an integral part of apprenticeship school training. Otherwise, most carpenters are destined to experience pain during a considerable portion of their working lifetime. This prognosis was especially supported by findings of the physical examination, which showed relatively high rates of electrodiagnosis of carpal tunnel syndrome $(14.3 \%)$, wrist tendinitis $(11.4 \%)$, and median mononeuropathy $(34.0 \%)$ of the wrist in the completely asymptomatic controls. In conclusion, there were numerous anecdotal 
comments by journeymen in their late 30 s and early 40 s expressing fears that their carpentry career would soon end due to the strains and pains currently experienced.

The study was funded by NIOSH.

1 Roto P, Kivi P. Prevalence of epicondylitis and tenosynovitis among meatcutters. Scand $\mathcal{f}$ Work Environ Health among meatcut $1991 ; 17: 32-7$.

2 Viikari-Juntura E, Kurppa K, Kuosma E, et al. Prevalence of epicondylitis and elbow pain in the meat-processing industry. Scand $\mathcal{f}$ Work Environ Health 1991;17:38-45.

3 Silverstein BA, Fine LJ, Stetson D. Hand-wrist disorders among investment casting plant workers. F Hand Surg $[\mathrm{Am}]$ 1987;12:838-44.

4 Kuorinka I, Koskinen P. Occupational rheumatic disease and upper limb strain in manual jobs in a light mechanical industry. Scand $\mathcal{F}$ Work Environ Health Suppl 1979;3:39-47.

5 Hales TR, Bernard BP. Epidemiology of work-related musculoskeletal disorders. Orthop Clin North Am 1996; 54:679-709.

6 Bernard B, Sauter S, Fine L, et al. Job task and psychosocial risk factors for work-related musculoskeletal disorders among newspaper employees. Scand $\mathcal{F}$ Work Environ Health 1994;20:417-26.

7 Bhattacharya A, Mueller M, Putz-Anderson V. Traumatogenic factors affecting knees of carpet installers. Appl togenic factors affecting

Ergon 1985;16:243-50.
8 US Department of Labor. Occupational injuries and illnesses in the United States by industry, 1990. Washington, DC: US Department of Labor, 1992. (Bureau of Labor Statistics Bulletin 2399.)

9 Atterbury MR, Limke J, Lemasters GK, et al. Nested case control study of hand and wrist work-related musculoskeletal disorders in carpenters. Am $\mathcal{F}$ Ind Med 1996;30 695-701.

10 Bhattacharya A, Greathouse WJL, Warren J, et al. An ergonomic walkthrough observation of carpentry tasks: a pilot study. Applied Occupational and Environmental Hygiene fournal 1997;12:278-87.

11 Armstrong TJ, Buckle P, Fine LJ, et al. A conceptual model for work-related neck and upper limb musculoskeletal disorders. Scand f Work Environ Health 1993;19:73-84.

$12 \mathrm{Hsiao} \mathrm{H}$, Stanevich RL. Injuries and ergonomic applications in construction. In: Bhattacharya A, McGlothlin JD, eds.
Occupational ergonomics: theory and applications. New York: Marcel Dekker, 1996:545-68.

13 Robinson CF, Petersen M, Sieber WK, et al. Mortality of carpenters' union members employed in the US construction or wood products industries, 1987-90. Am f Ind Med 1996;30:674-94.

14 Ohio Bureau of Worker's Compensation. Ohio carpenters in the private sector construction industry -1990. Injury/ illness statistics. Columbus, OH: OBWC, 1990.

15 Lemasters GK, Atterbury MR. The design and evaluation of a musculoskeletal and work history questionnaire in occupation. In: Bhattacharya A, McGlothin, JD, eds. Occupational ergonomics. theory and applications. New York: Marcel Dekker, 1996:431-62.

16 Anderson KO, Bradley LA, Young LD, et al. Rheumatoid arthritis: review of psychological factors related to etiology effects and treatment. Psychol Bull 1986;98:358-87.

17 Karasek R. Control in the workplace and its health-related aspects. In: Sauter S, Hurrell J, Cooper C, eds. Fob control and worker health. New York: Wiley, 1989:130.

18 Leino, P. Symptoms of stress predict musculoskeletal disorders. F Epidemiol Community Health 1989;43:293-300.

19 Hurrell J, McLaney M. Exposure to job stress-a new psychometric instrument. Scand F Work Environ Hlth 1984;4: $27-8$.

20 Thun $M$, Tanaka S, Smith AB, et al. Morbidity from repetitive knee trauma in carpet and floor layers. $\mathrm{Br} \mathcal{F}$ Ind $\mathrm{Med}$ 1987;44:611-20.

21 Schelessman JJ. Sample size requirements in cohort and case-control studies of disease. Am $\mathcal{F}$ Epidemiol 1974;99: 381-4.

22 Harrell FE Jr, Lee KL, Mark DB. Multivariable prognostic models: issues in developing models, evaluating assumptions and adequacy, and measuring and reducing errors. Stat Med 1996;15:361-87.

23 Booth-Jones, AD, Lemasters GK, Succop P, et al. Reliability of questionnaire information measuring musculoskeletal symptoms and work histories. Am Ind Hyg Assoc f 1998;59: 20-4.

24 Bhattacharya A, Greathouse L, Warren J, et al. An ergonomic walkthrough observation of carpentry tasks: a pilot study. Appl Occup Environ Hyg 1997;12:278-87.

25 Morata T, Lemasters G. Epidemiologic considerations when evaluating occupational hearing loss. State of the art review. Philadelphia: Hanley and Belfus, 1995.

26 Moran JB. Ergonomic awareness for carpenters: overview. Appl Occup Environ Hyg 1995;10:734-6. 